\title{
Insuficiencia cardíaca en hospitales chilenos: resultados del Registro Nacional de Insuficiencia Cardíaca, Grupo ICARO
}

\author{
Pablo Castro G, José Luis Vukasovic R, Eduardo G arcés S, \\ Luis Sepúlveda M, Marcela Ferrada K, Sergio Alvarado 0 , en \\ representación de grupo ICARO (Insuficiencia Cardíaca: \\ Registro y 0 rganización).
}

\section{Cardiac failure in Chilean hospitals: results of the National Registry of Heart Failure, ICARO}

Background: Heart failure (HF) is a major public health problem. In Chile hospitalized patients due to HF have not been characterized. Aim: To evaluate clinical profile and outcome of patients hospitalized for heart failure in Chilean hospitals. Patients and Methods: Prospective registry of 14 centers. Patients hospitalized for HF in functional class III and IV were included. Epidemiological and clinical data, functional class, type of presentation, decompensation cause, electrocardiogram, echocardiogram, treatment and evolution were registered. Results: Three hundred seventy two patients aged $69 \pm 13$ years old, $59 \%$ men, were assessed. The main etiologies of $\mathrm{HF}$ were ischemic in $31.6 \%$, hypertensive in $35.2 \%$, valvular in $14.9 \%$ and idiopathic in $7.4 \%$. There was a history of hypertension $69 \%$, diabetes in $35 \%$, myocardial infarction in $22 \%$, atrial fibrillation (AF) in $28 \%$. The presentation form of $\mathrm{HF}$ was chronic decompensated in $86 \%$, acute in $12 \%$, refractory in $2 \%$. The causes of decompensation were non compliance with diet or medical prescriptions in 28\%, infections in 22\% and AF 17\%. ECG showed AF in 36\% and left bundle branch block in $16 \%$. Echocardiography was performed in $52 \%$ of the patients, $69 \%$ had left ventricular ejection fraction $<40 \%$. On admission, $39 \%$ received angiotensin converting enzyme (ACE) inhibitors, $15 \%$ beta-blocker, $25 \%$ digoxin, $16 \%$ spironolactone and $53 \%$ furosemide. The mean hospital stay was $11 \pm 10$ days and mortality was $4.5 \%$. Conclusions: The elderly is the age group most commonly admitted to hospital due to HF. The main etiologies were ischemic and hypertensive. The main causes for decompensations were noncompliance with diet or medical prescriptions and infections. A significant proportion had a relatively well preserved ventricular systolic function (Rev Méd Chile 2004; 132: 655-62).

(Key Words: Cardiac output, low; Heart diseases; Heart failure, congestive; Myocardial ischemia)

Recibido el 8 de enero, 2004. Aceptado el 7 de abril, 2004.

Departamento Estudios Multicéntricos Sociedad Chilena de Cardiología

Correspondencia a: Dr. Pablo Castro. Departamento Enfermedades Cardiovasculares. Pontificia Universidad Católica de Chile, Marcoleta 347, Santiago, Chile.

E-mail: pcastro@med.puc.cl 
T a insuficiencia cardíaca (IC) constituye actualmente un importante problema de salud públi$\mathrm{ca}^{1-3}$. En países desarrollados, la prevalencia e incidencia de esta enfermedad ha aumentado en forma progresiva. Probablemente han contribuido a este fenómeno la mayor expectativa de vida, los mejores tratamientos y la mayor sobrevida de otras enfermedades cardiovasculares. La situación descrita se asocia a una elevada mortalidad, necesidad de hospitalizaciones, deterioro en la calidad de vida y consumo de recursos s $^{1,2,4,5}$.

La IC se considera actualmente un prototipo de enfermedad crónica que afecta a pacientes ancianos, que demandan habitualmente una atención multidisciplinaria de distintos profesionales de la salud. La IC constituye uno de los principales motivos de hospitalización en países desarrollados como Estados Unidos y España ${ }^{2,6}$. La práctica clínica en la IC difiere llamativamente entre países, entre áreas geográficas de un mismo país y entre diferentes subgrupos de pacientes ${ }^{1,3,5}$. Con frecuencia estas diferencias en procedimientos de diagnóstico y medidas terapéuticas dependen de las peculiaridades de cada organización de salud ${ }^{7,8}$. También dependen de las diferencias en las características de los pacientes incluidos en los ensayos clínicos y registros de los que requieren hospitalización. Este hecho puede explicar por qué evidencias de la eficacia de los distintos fármacos expresadas en guías clínicas, no corresponden necesariamente a la práctica clínica ${ }^{9}$.

En nuestro país no existe información sobre las características de los pacientes hospitalizados por IC. Con el propósito de dar respuesta a estas interrogantes, la Sociedad Chilena de Cardiología constituyó, el año 2001, un grupo de trabajo en IC que, entre otros objetivos, se propuso analizar las características clínicas, tratamiento y evolución intrahospitalaria de los pacientes ingresados en hospitales chilenos con el diagnóstico de IC descompensada. Esta información contribuirá a conocer la realidad nacional e identificar aquellos aspectos concretos de nuestra práctica clínica susceptibles de mejorar.

\section{PaCientes y MÉTodo}

Desde enero de 2002, en 14 centros chilenos, se recolectó información prospectiva de 372 pacien- tes hospitalizados por IC CF III o IV. El criterio diagnóstico fue: síntomas de IC y evidencias objetivas de disfunción cardíaca objetivada en el ecocardiograma o la radiografía de tórax; en casos dudosos se consideró la respuesta al tratamiento dirigido a la $\mathrm{IC}^{10}$. Se excluyeron todos los pacientes en los cuales el motivo principal de hospitalización no fue la IC y aquellos pacientes que estando previamente asintomáticos y sin antecedente de IC previa presentaron IC o shock cardiogénico secundario a un cuadro coronario agudo. En una ficha especialmente diseñada se registró: características demográficas, co-morbilidad, previsión, nivel educacional, antecedentes de hospitalizaciones previas por IC, etiología de la cardiopatía de base, factores de descompensación de la hospitalización actual, hallazgos del electrocardiograma y ecocardiograma, incluida la fracción de eyección de ventrículo izquierdo (FEVI). Se consignó el tratamiento al ingreso, alta y evolución intrahospitalaria.

La IC se clasificó de acuerdo a su presentación como: crónica descompensada cuando existían síntomas de más de 3 meses de evolución y agravación en las últimas 2 semanas, IC aguda cuando los síntomas aparecen en los últimos 2 meses y el cuadro clínico y la ecocardiografía sugieren una etiología aguda y como IC refractaria cuando existía persistencia de síntomas severos a pesar del uso de una terapia médica optimizada. Las historias clínicas correspondientes, incluyendo radiografías de tórax, exámenes de laboratorio y ecocardiogramas fueron revisadas por el equipo investigador de cada centro. Las fichas de los centros correspondientes fueron enviadas a la Sociedad Chilena de Cardiología, donde una enfermera especializada revisó cada ficha clínica antes de ser ingresada a una base de datos especialmente diseñada.

Estadística: Se realizó en el centro de estadística de la Escuela de Salud Pública de la Universidad de Chile utilizando el sistema SPSS. Las variables continuas son expresadas como promedio \pm desviación estándar. En la comparación de variables continuas entre el ingreso y egreso se utilizó la prueba de $t$ de Student, las proporciones de usos de distintos medicamentos se comparó mediante la prueba de $\chi^{2}$. Un valor de $\mathrm{p}<0,05$ se consideró significativo. 


\section{Resultados}

Características demográficas. En 14 centros chilenos se recolectó información de 372 pacientes. El $59 \%$ fueron hombres, la edad promedio fue de $69 \pm 13$ años, correspondiendo $78 \%$ a pacientes mayores de 60 años y $22 \%$ a mayores de 80 años (Figura 1). La distribución por sexo fue similar entre los diferentes rangos de edad. Entre las patologías más relevantes se encontró hipertensión arterial en $69 \%$ de los casos, diabetes mellitus en $35 \%$, tabaquismo en $16 \%$, infarto previo en $22 \%$ y enfermedad pulmonar obstructiva crónica en $8 \%$ de los pacientes. El 28\% de los pacientes tenía antecedente de fibrilación auricular y $8 \%$ de accidente vascular encefálico (Tabla 1).

Previsión y nivel educacional. La previsión fue Fonasa en $73,3 \%$ e Isapre en 19,2\%. El 7,6\% de los pacientes no tenía previsión. El $61 \%$ tenía educación básica, $17 \%$ estudios superiores y $9 \%$ era analfabeto (Figura 2).

Etiología de la cardiopatía de base. Las principales etiologías de la IC fueron cardiopatía hipertensiva en $35 \%$ de los pacientes, enfermedad coronaria en 32\%, enfermedad valvular en $15 \%$ y miocardiopatía dilatada idiopática en 7,4\% de los pacientes (Tabla 2).

\section{Tabla 1. C aracterísticas demográficas y antecedentes de los pacientes}

\begin{tabular}{|lr|}
\hline Edad (años) & $69 \pm 13$ \\
Hombres (\%) & 59 \\
Antecedentes & \\
- Hipertensión arterial (\%) & 69 \\
- Diabetes Mellitus (\%) & 35 \\
- Tabaquismo (\%) & 16 \\
- Infarto al miocardio (\%) & 22 \\
- Angina crónica (\%) & 10 \\
- Cirugía de revascularización coronaria (\%) & 9,4 \\
- Angioplastia coronaria (\%) & 0,8 \\
- Fibrilación auricular (\%) & 35 \\
- Enfermedad pulmonar obstructiva crónica (\%) & 8 \\
- Accidente vascular encefálico (\%) & 8 \\
Previsión & \\
- FONASA (\%) & 73 \\
- ISAPRE (\%) & 19 \\
- Sin previsión (\%) & 8 \\
\hline
\end{tabular}

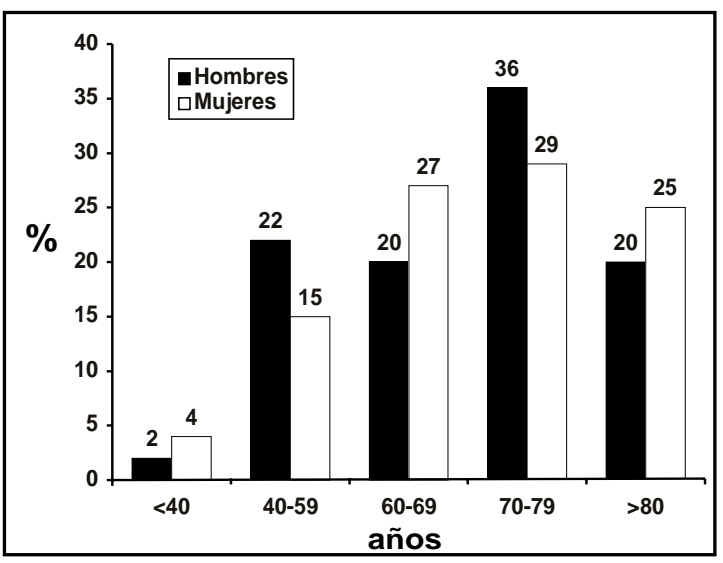

Figura 1. Distribución por edad y sexo de los pacientes.

Características de la IC y forma de presentación. La mayor proporción de pacientes correspondió a IC crónica descompensada (86\%), el $12 \%$ a IC aguda y $2 \%$ a IC refractaria (Figura 3). La mayoría de los pacientes presentó evidencias de congestión pulmonar clínica o radiológica $(87,8 \%)$ y congestión visceral (27\%). Un bajo porcentaje de los casos se presentó con hipotensión $(5,9 \%)$ o en shock cardiogénico (1,1\%) (Figura 4).

Factores precipitantes de la hospitalización actual y hospitalizaciones previas por IC (Tabla 3). La principal causa de descompensación fue la falta de adherencia a la dieta, medicamentos o a ambos (28\%), seguida de infecciones (22\%), fundamentalmente respiratorias y la fibrilación auricular (17\%). Una menor proporción correspondió a crisis hipertensiva y a cuadros coronarios agudos.

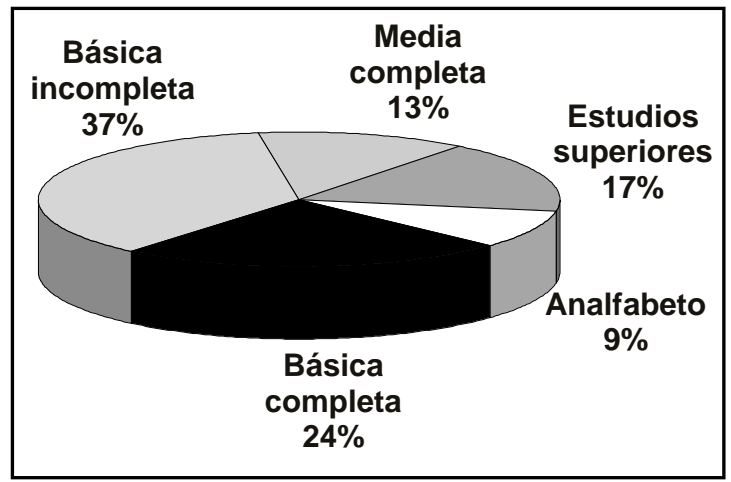

FIGURA 2. Nivel educacional de los pacientes hospitalizados por IC. 
El 35\% de los pacientes tenía antecedentes de hospitalización previa por IC; de éstos, $12 \%$ ocurrieron en el mes previo al ingreso actual, $16 \%$ entre 13 meses, 30\% entre 3-12 meses y 42\% más de 1 año.

Características del electrocardiograma de ingreso. El 55\% de los pacientes estaba en ritmo sinusal, $36 \%$ en fibrilación auricular y 9\% en ritmo de marcapaso. Se encontró imagen de bloqueo completo de rama izquierda en $16 \%$, bloqueo completo de rama derecha en $8 \%$ y secuela de necrosis miocárdica en $17 \%$. La duración promedio del QRS fue de $107 \pm 31 \mathrm{msec}$.

Ecocardiograma y función ventricular. Al 52\% de los pacientes se les realizó ecocardiograma. El diámetro diastólico promedio fue de $60,7 \pm 12 \mathrm{~mm}$, el diámetro sistólico $48 \pm 13 \mathrm{~mm}$ y el diámetro de la aurícula izquierda $47 \pm 9,8 \mathrm{~mm}$. La FEVI promedio fue de $35 \pm 15 \%$. La FEVI fue $\leq 40 \%$ en $69 \%$ de los pacientes (Figura 5).

Tabla 2. Etiología de la IC

\begin{tabular}{|lrc|}
\hline Etiología & $\mathrm{n}$ & $\%$ \\
\hline Hipertensiva & 123 & 35 \\
Isquémica & 111 & 31,6 \\
Valvular & 52 & 14,8 \\
Idiopática & 27 & 7,4 \\
Hipertensiva + isquémica & 15 & 4,3 \\
Alcohólica & 8 & 2,2 \\
Hipertrófica & 5 & 1,4 \\
Otra & 10 & 2,8 \\
\hline
\end{tabular}

Intervenciones intrahospitalarias. El 19\% de los pacientes requirió uso de inótropos IV, 21,6\% vasodilatadores IV, $4 \%$ ventilación mecánica y $2,2 \%$ diálisis. Se realizó monitoreo hemodinámico con catéter de Swan-Ganz en 4,9\% de los pacientes, revascularización coronaria en 1,6\% y cirugía de recambio valvular en $0,8 \%$.

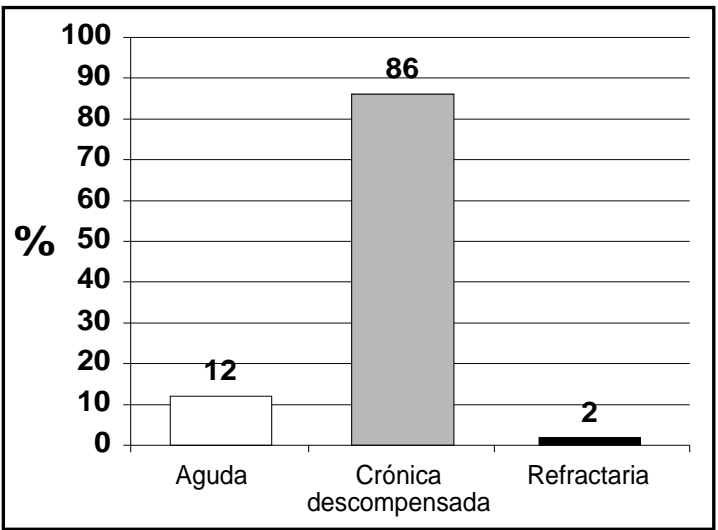

FIgURA 3. Clasificación clínica de la IC.

Tabla 3. Factores precipitantes de la hospitalización (\%)

Falta adherencia dieta/medicamentos 28

Sin causa identificable $\quad 26$

Fibrilación auricular $\quad 17$

Neumonía 15

Crisis HTA 10

Angina inestable/IAM 9

Otras infecciones $\quad 7$

Otras arritmias 4

Taquicardia ventricular $\quad 0,8$

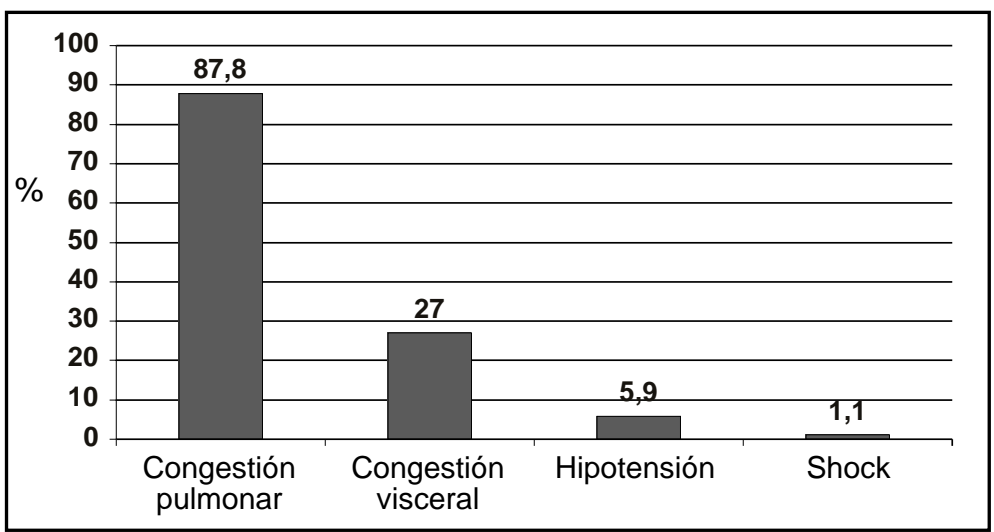

FIGURA 4. Formas de presentación clínica de la IC. 
Tabla 4. Evolución de exámenes de laboratorio y signos vitales

\begin{tabular}{|lccc|}
\hline & Ingreso & Alta & Valor $\mathrm{p}$ \\
\hline Peso $(\mathrm{kg})$ & $77,2 \pm 66$ & $68,3 \pm 14$ & $<0,045$ \\
PAS (mmHg) & $135,7 \pm 35$ & $118,8 \pm 19,3$ & $<0,001$ \\
PAD (mmHg) & $82,0 \pm 21$ & $68,8 \pm 13,2$ & $<0,001$ \\
FC (l/min) & $93,0 \pm 30$ & $75,3 \pm 12,3$ & $<0,001$ \\
Hematocrito (\%) & $39,0 \pm 7,0$ & $37,0 \pm 10,1$ & 0,685 \\
N ureico (mg/dL) & $30,1 \pm 17,3$ & $32,0 \pm 18,3$ & 0,502 \\
Creatinina (mg/dL) & $1,6 \pm 1,23$ & $1,5 \pm 1,01$ & 0,549 \\
Sodio plasmático (mEq/L) & $139,1 \pm 21,7$ & $134,8 \pm 20,3$ & 0,624 \\
Potasio plasmático (mEq/L) & $4,5 \pm 3,3$ & $4,4 \pm 3,19$ & 0,963 \\
\hline
\end{tabular}

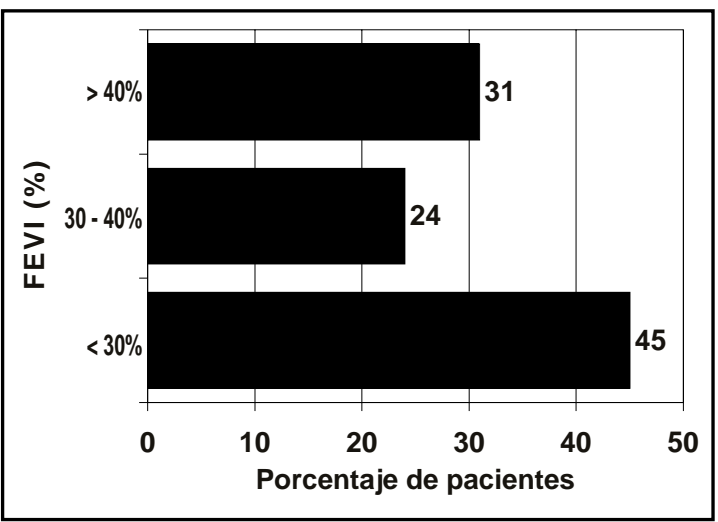

FIgURA 5. Distribución de las determinaciones de fracción de eyección ventricular izquierda.

Evolución clínica y de exámenes de laboratorio. Durante la estadía hospitalaria hubo una disminución significativa del peso corporal (promedio 8 $\mathrm{kg}$ ), la presión arterial sistólica y diastólica disminuyeron en promedio 17 y $14 \mathrm{mmHg}$ respectivamente $(135 / 82$ a $118 / 68 \mathrm{mmHg})$ y la frecuencia cardíaca promedio se redujo de $93 \pm 30$ a $75 \pm 12$ latidos/min. La creatinina al ingreso fue de $1,6 \pm 1,2 \mathrm{mg} / \mathrm{dL}$ y no se modificó durante la hospitalización. No se observaron cambios significativos en los valores de sodio y potasio plasmático (Tabla 4).

La estadía hospitalaria fue de $11 \pm 10$ días. La mortalidad intrahospitalaria fue de $4,5 \%$ y en $87,3 \%$ de los pacientes se observó una mejoría clínica significativa.

Tratamiento. En la Tabla 5 se muestra el tratamiento al ingreso y alta de los pacientes. Al ingreso el 39\% utilizaba inhibidores de la enzima convertidora, en
Tabla 5. M edicamentos al ingreso y alta en pacientes hospitalizados por IC

\begin{tabular}{|lccc|}
\hline & $\begin{array}{c}\text { Ingreso } \\
\%\end{array}$ & $\begin{array}{c}\text { Alta } \\
\%\end{array}$ & Valor p \\
\hline IECA & 39 & 55 & $<0,001$ \\
Captopril & 4,6 & 6,7 & \\
Enalapril & 31,9 & 47,1 & \\
Otro & 2,1 & 1 & \\
Betabloqueador & 15 & 21 & 0,019 \\
Carvedilol & 5,6 & 12,9 & \\
Propanolol & 3,8 & 5,6 & \\
Otro & 4,3 & 2,4 & \\
Antagonista RA II & 5,5 & 3,7 & 0,008 \\
Losartan & 3,8 & 3,2 & \\
Otro & 1,9 & 0,5 & \\
Hidralazina & 8,6 & 12,9 & 0,15 \\
Nitratos & 23,1 & 25,5 & 0,36 \\
Digoxina & 25 & 34,1 & $<0,001$ \\
Furosemida & 53 & 74,8 & $<0,001$ \\
Espironolactona & 16 & 36,2 & $<0,001$ \\
Bloqueador del calcio & 14 & 10,5 & 0,009 \\
Amiodarona & 14,3 & 23,6 & $<0,001$ \\
Aspirina & 21,7 & 44 & $<0,001$ \\
Anticoagulante & 23,9 & 16,6 & 0,002 \\
\hline
\end{tabular}

IECA = Inhibidor de la enzima convertidora de angiotensina, RA II= Receptor de la angiotensina II.

este grupo el fármaco más empleado fue el enalapril, $15 \%$ beta bloqueadores, mayoritariamente carvedilol, $16 \%$ espironolactona, 53\% furosemida, 5,5\% antagonistas de los receptores de la angiotensina II, 25\% digital, 10\% bloqueadores de los canales de calcio y $14 \%$ amiodarona. Al egreso existió un incremento 
significativo en la proporción de pacientes que recibían inhibidores de la enzima convertidora de angiotensina, betabloqueadores, espironolactona, digital diuréticos y amiodarona, con disminución del empleo de bloqueadores de los canales de calcio. La proporción de pacientes que recibía otros vasodilatadores como hidralazina y nitratos no se modificó.

\section{Discusión}

En Chile, la hospitalización por IC ocurre en una población de edad avanzada, siendo las principales etiologías la hipertensión arterial y la enfermedad coronaria. Esto es similar a lo observado en otros países como Estados Unidos, Argentina y España. Sin embargo, a diferencia de Estados Unidos, en Chile como en España y Argentina la patología valvular continúa constituyendo una etiología importante de $\mathrm{IC}^{11-13}$.

La edad de los pacientes incluidos en el registro fue con una alta proporción de pacientes mayores de 60 años, un leve predominio de sexo masculino y una elevada concomitancia de otras enfermedades. Este hallazgo coincide con lo observado en otros registros, como el español, francés y argentino ${ }^{7,12-13}$. Sin embargo, difiere de las características de los pacientes incluidos en la mayoría de los ensayos clínicos en los que la edad media de los pacientes es menor a los 60 años ${ }^{14-17}$. Es así como muchos de los pacientes de nuestro registro no habrían sido incluidos en estos estudios.

La coexistencia de otras enfermedades en los pacientes hospitalizados por IC tiene gran relevancia clínica e influye en el pronóstico y tratamiento de estos pacientes ${ }^{18}$. Un gran número de nuestros pacientes tiene antecedentes de diabetes mellitus, hipertensión arterial u otras co-morbilidades, lo que en ocasiones requiere de un manejo multidisciplinario que en algunas circunstancias es tan importante como el de la IC ${ }^{19}$

Otro hallazgo relevante fue el bajo nivel de escolaridad de los pacientes de nuestro registro. Esta situación puede estar determinada por la presencia de centros que reciben pacientes de áreas rurales y la participación de un gran número de pacientes de edad avanzada. El nivel educacional junto al económico, probablemente, son factores determinantes en la falta de adherencia a las indicaciones médicas, mal cumplimiento del tratamiento, tórpida evolución clínica y una mayor necesidad de re-hospitalizaciones. Este hecho debe ser considerado en la elaboración de programas de seguimiento e intervención en esta población, que presenta una alta incidencia de re-hospitalizaciones y donde la causa más frecuente de descompensación fue la falta de adherencia a dieta 0 medicamentos ${ }^{20}$.

Luego de la falta de adherencia al tratamiento y de las infecciones, la fibrilación auricular fue la causa de descompensación más frecuente en este grupo. Su presencia está relacionada probablemente al envejecimiento de la población y a la presencia de hipertensión arterial. La fibrilación auricular es un fenómeno conocido de descompensación, tanto en pacientes con disfunción sistólica como diastólica ${ }^{21}$.

Desde el punto de vista de diagnóstico, nosotros utilizamos los criterios de la Sociedad Europea de Cardiología $^{10}$. En nuestro registro la presencia de congestión pulmonar y de cardiomegalia en la radiografía de tórax, asociada a síntomas de IC, permitía la inclusión en el registro, aun sin disponer de un ecocardiograma. El 52\% de los pacientes contaba con un ecocardiograma durante la hospitalización, este porcentaje de ecocardiograma es inferior al comunicado en otros registros ${ }^{7,22}$. Las guías europeas insisten en la necesidad de un diagnóstico con valoración objetiva de la función cardíaca con ecocardiografía, por lo general, para evitar diagnósticos imprecisos. En algunos casos, el uso exclusivo del cuadro clínico asociado a una radiografía de tórax concordante con insuficiencia cardíaca nos permitió la participación de un mayor número de centros, obteniendo de esta manera una mejor representación de la realidad nacional de esta patología. Sin embargo, este hecho debe hacer reflexionar sobre las limitaciones existentes en el medio nacional para precisar el diagnóstico de esta entidad.

Dentro del grupo de pacientes con diagnóstico ecocardiográfico, un porcentaje importante presentaba una FEVI $>40 \%$ (31\%), lo que puede estar en relación a la presencia de cardiopatía hipertensiva y disfunción diastólica. Estos resultados son similares a los reportados en otros estudios ${ }^{7,12}$, sin embargo, la dificultad en el diagnóstico de la disfunción diastólica e identificar en forma precisa a este grupo de pacientes, puede llevar a un diagnóstico errado de IC. 
En el registro llama la atención la alta utilización de inótropos, particularmente si consideramos que la mayor parte de los pacientes hospitalizados tenían historia de IC crónica con evidencias de congestión pulmonar o visceral y sólo una minoría se presentó con hipotensión 0 shock. Estos antecedentes hacen razonable postular que muchos de estos pacientes pudieran haberse tratado solamente con optimización del tratamiento vasodilatador o depletivo. Esta consideración es relevante, dado que estudios recientes han cuestionado el uso rutinario de inótropos en pacientes hospitalizados por IC ${ }^{23}$.

El análisis del tratamiento farmacológico al ingreso demuestra que en nuestro medio aún existe una baja utilización de inhibidores de la enzima convertidora de angiotensina y beta bloqueadores, por otra parte, se demuestra que vasodilatadores tradicionales como la hidralazina y nitratos continúan formando parte del armamentanio terapéutico. A su vez, es interesante el porcentaje de pacientes que utiliza espironolactona. Durante la hospitalización existió una optimización del tratamiento con un mayor empleo de terapias que han demostrado un beneficio sobre la morbimortalidad de los pacientes con IC. Así por ejemplo, al egreso se incrementó la proporción de pacientes que utilizaban inhibidores de la enzima convertidora de angiotensina, de beta bloqueadores y de espironolactona, y disminuyó el empleo de bloqueadores del calcio cuya eficacia no ha sido demostrada, a lo menos en pacientes con disfunción sistólica. El empleo de los antagonistas de los receptores de la angiotensina II en el registro fue más bien discreto. A pesar de la optimización de la terapia durante la hospitalización, aún consideramos que el empleo de algunos de estos fármacos se encuentra por debajo de lo ideal o de otros registros ${ }^{12,13}$. La presencia de insuficiencia renal no pareciera ser el motivo principal para la limitada utilización de inhibidores de la enzima convertidora ya que solo $15 \%$ de nuestra población presentaba valores de creatinina $>2 \mathrm{mg} / \mathrm{dL}$ Al egreso también existió un aumento en el uso de amiodarona, digital, diuréticos y aspirina con disminución del uso de

\section{REFERENCIAS}

1. Heart Failure: evaluation and care of patients with left ventricular dysfunction. US Department of Health and Human Services. Publication № 940612, Maryland, 1994. anticoagulantes orales. Este último hecho ocurre a pesar de la alta prevalencia de fibrilación auricular y puede estar dado por la reticencia a utilizar anticoagulantes orales en una población de pacientes de edad avanzada o de ruralidad significativa.

A pesar de la edad avanzada de nuestros pacientes la mortalidad intrahospitalaria de esta serie fue baja $(4,5 \%)$ e inferior a la reportada en otros registros de la literatura ${ }^{12,13,24}$.

Limitaciones del estudio. Este registro tiene limitaciones porque no comprende a todos los pacientes hospitalizados por IC en Chile. Sin embargo, tiene el valor de integrar centros de distinta complejidad, siendo la mitad de ellos del área metropolitana y el resto de otras regiones del país. Otro posible sesgo en la selección puede estar dado por la participación de centros en los cuales existen profesionales interesados y posiblemente con una mayor experiencia en el manejo de la IC. La limitación en la disponibilidad de ecocardiografía ha sido ya comentada con las dificultades en la evaluación de la disfunción diastólica. A pesar de estas limitaciones pensamos que estos hallazgos preliminares son valiosos y representan el espectro de esta patología en nuestro medio y enfatizan las dificultades en la certificación diagnóstica y la necesidad de mejonías en el manejo de este síndrome. El seguimiento de estos pacientes, intervenciones dirigidas y el análisis de la evolución de las terapias en nuestro medio podńan contribuir al progreso en el manejo de esta patología. En este sentido, a futuro, la comparación de los resultados del registro en dos peńodos parece ser relevante.

En resumen, en Chile la hospitalización por IC ocurre en una población de edad avanzada, con una elevada asociación a co-morbilidades. A nivel nacional es importante objetivar el diagnóstico, realizar un manejo multidisciplinario, intervenciones educativas que faciliten la adherencia al tratamiento y optimizar la calidad de la terapia, para mejorar la calidad de vida, retardar la progresión del daño miocárdico y reducir en definitiva los requerimientos de hospitalización.

2. Rodríguez Artalejo F, Guallar-Castimón P, Banegas R, DeL REY J. Trends in hospitalization and mortality for heart failure in Spain. Eur Heart J 1997; 18: 1537-40.

3. SHARPE N. Management principles: much more to be gained. En: Heart failure management. London: Martin Dunitz, 2000. 
4. The Task Force on Heart Failure of the European Society of Cardiology. Guidelines for the diagnosis of heart failure. Eur Heart J 1995; 16: 741-51.

5. Hobbs FDR, Davis RC, McLeod S, Marshall T, Kenkre J, LANCASHIRE R. Prevalence of Heart Failure in high risk groups. J Am Coll Cardiol 1998; 31: 5-85.

6. ERHaRdt L, Cune C. Community Management of Heart Failure 2000. En: Heart failure management. London: Martin Dunitz, 2000.

7. Cohen-Solal A, Desnos M, Delahaye F, Emerian JP, Hanania G. A national survey of heart failure in French hospitals. Eur Heart J 2000; 21: 763-9.

8. Cowie MR, Mosterd A, Wood DA, Deckers JW, Poole-Wilson PA, Sutton GC et al. The epidemiology of heart failure. Eur Heart J 1997; 8: 208-25.

9. Philbin EF, ANdreou C, Rocco TA, Lynch LJ, BaKer SL Patterns of angiotensin-converting enzyme inhibitor use in congestive heart failure in two community hospitals. Am J Cardiol 1996; 77: 832-8.

10. European Society of Cardiology: WJ Remme and K Swedberg, Task Force for the Diagnosis and Treatment of Chronic Heart Failure. Eur Heart J 2001; 22: 1527-60.

11. Polanczy CA, Newton C, Dec GW, Di Salvo TG. Quality of care and hospital readmision in congestive heart failure: An explicit review process. J Cardiac Failure 2001; 7: 289-98.

12. Grupo de Trabajo de Insuficiencia Cardíaca de la Sociedad Española de Medicina Interna (SEMI). La insuficiencia cardíaca en los servicios de medicina interna (estudio SEMI-IC). Med Clin (Barc) 2002; 118: 605-10.

13. Thierer J, Iglesias D, Ferrante D, Marino J, Diez M, RoLong B ET AL A. Registro nacional de internación por insuficiencia cardíaca. Factores responsables, evolución hospitalaria y predictores de mortalidad. Revista Argentina de Cardiología 2002; 70: 261-73.

14. The Digitalis Investigation Group. The effect of digoxin on mortality and morbidity in patients with heart failure. N Engl J Med 1997; 336: 525-33.

15. THE CONSENSUS TRIAL STudY GROUP. Effects of enalapril on mortality in severe congestive heart failure. Results of the cooperative north Scandinavian Enalapril Survival Study. NEngJ Med 1987; 316: 1429-35.

16. Pitt B, Zannad F, Cody R, Castaigne A, Pérez A, PALENSKY J. The effect of spironolactone on morbidity and mortality in patients with severe heart failure. N Engl J Med 1999; 341: 709-17.

17. CIBIS II Investigators and CommitTeEs. The cardiac insufficiency bisoprolol study (CIBIS-II): a randomized trial. Lancet 1998; 353: 9-13.
18. Forteza-Rey J. Comorbilidad e insuficiencia cardíaca. Rev Clin Esp 2001; 201 (Supl 1): 16-21.

19. Kasper EK, Gernstenbltit G, Hefter G, Van Anden E, Brinker JA, ThiEMann DR et al. A randomized trial of the efficacy of multidisciplinary care in heart failure outpatients at high risk of hospital readmission. J Am Col Cardiol 2002; 39: 471-80.

20. Philbin EF, Dec GW, Jenkins PL, DiSalvo TG. Socioeconomic status as an independent risk factor for hospital readmission for heart failure. Am J Cardiol 2001; 87: 1367-71.

21. Tsuyuki RT, McKelvie RS, Arnold MO, Avezum A, Barretto AC, Carvalho AC et al. Acute Precipitants of Congestive Heart Failure Exacerbations. Arch Intern Med 2001; 161: 2337-42.

22. Clarke K, Gray D, Hampton J. Evidence of inadequate investigation and treatment of patients with heart failure. B Heart J 1994; 71: 584-7.

23. Cuffe MS, Califf RM, Adams KF, Benza R, Bourge R, Colucci WS Et AL. Outcomes of a Prospective Trial of Intravenous Milrinone for Exarcebations of Chronic Heart Failure (OPTIME-CHF) Investigators. JAMA 2002; 287: 1578-80.

24. BeLotti P, Badano LP, Acquarone N, GriLo R, LoPinto G, MagGioni AP. Speciality related differences in the epidemiology, clinical profile, management and outcome of patients hospitalized for heart failure. Eur Heart J 2001; 22: 596-604.

Lista de centros e investigadores participantes grupo ICARO: Clínica Las Condes: Dr. Ronald Kauffmann; Hospital Regional La Serena: Dr. Carlos Echevemá, EU Alejandra Montes; Hospital Regional de Talca: Drs. Ennique Mercadal, Claudio Pacheco; Hospital Regional de Valdivia: Drs. Eduardo Garcés, Germán Eggers, EU Pilar Muñoz; Hospital Base Puerto Montt: Dr. Jaime Venegas; Hospital Clínico Universidad Católica: Dr. Pablo Castro, EU Marcela Marchant; Hospital Clínico Universidad de Chile: Dr. Luis Sepúlveda; Hospital del Salvador. Drs. José Luis Vukasovic, Víctor Rossel; Hospital San Juan de Dios: Dr. Juan Ramón Soto; Hospital Barros Luco: Drs. Crisólogo Venegas, Patricio Yovaniniz; Hospital Naval de Viña del Mar: Dr. Fernando Cárdenas; Hospital Regional de Antofagasta: Dr. Manuel Saavedra; Hospital Regional de Temuco: Dr. Femando Lanas; Hospital Regional Punta Arenas: Dr. Guillermo Araneda.

Agradecimientos a Laboratorio Roche por la colaboración en la confección de fichas de registro y cooperación a la Sociedad Chilena de Cardiología para la organización del grupo ICARO. 